\title{
The Spectrum of Clinical and Urodynamic Findings in Patients with Spinal Tuberculosis Exhibiting Lower Urinary Tract Symptoms, before and after Spinal Surgical Intervention with Antitubercular Treatment: A Prospective Study
}

\author{
Nitin Shrivastava ${ }^{1}$, Prabhjot Singh ${ }^{1}$, Brusabhanu Nayak ${ }^{1}$, Bhavuk Garg ${ }^{2}$ \\ ${ }^{I}$ Department of Urology, All India Institute of Medical Sciences, New Delhi, India \\ ${ }^{2}$ Department of Orthopedics, All India Institute Of Medical Sciences, New Delhi, India
}

\begin{abstract}
Study Design: Observational study.
Purpose: This study aims to assess the clinical and urodynamic parameters in patients with spinal tuberculosis (TB) exhibiting lower urinary tract symptoms (LUTS) at the time of presentation and after spinal surgical intervention.

Overview of Literature: Variable urodynamic findings in patients with spinal TB.

Methods: We prospectively evaluated 10 patients with spinal TB exhibiting LUTS. Urinary symptoms were assessed by the American Urological Association (AUA) symptom score. We performed a urodynamic study (UDS), including electromyography, in all patients before and 3 months after spinal surgery.

Results: The mean age of patients was 29.7 years (range, 15-52 years), and the mean AUA symptom score was 12.5 and 11.8 before and after spinal surgery, respectively. Overall, five patients exhibited improvement in the AUA symptom score, and three showed no change, while two patients' condition worsened. We observed detrusor overactivity (DO) in two patients, and detrusor sphincter dyssynergia (DSD) in four patients. In addition, high-pressure voiding (HPV) was noted in two patients. On follow-up after spinal surgery, DO and DSD exhibited no improvement. Although HPV resolved, two patients developed new-onset poor compliance with worsening DO and DSD. Furthermore, two patients had bilateral hydronephrosis before surgery, which resolved on follow-up.

Conclusions: Patients with spinal TB exhibiting LUTS can display a spectrum of clinical presentations and variable UDS findings. As two patients exhibited new onset poor compliance with bilateral hydronephrosis in one of them, this study concludes that a close follow-up for upper tracts in these patients is required despite successful spinal surgery.
\end{abstract}

Keywords: Spinal tuberculosis; Urodynamics; Detrusor

\section{Introduction}

Spinal tuberculosis (TB) is a common health-related prob- lem in developing countries but is becoming a serious concern in developed nations as well. At present, TB is a global health-related problem. Osteoarticular TB accounts

Received Aug 26, 2018; Revised Nov 20, 2018; Accepted Dec 18, 2018

Corresponding author: Prabhjot Singh

Department of Urology, All India Institute of Medical Sciences, Ansari Nagar, New Delhi, 110029, India

Tel: +91-11-26594884, Fax: +91-11265841, 26588663, E-mail: drprabhjotsingh@gmail.com 
for approximately $20 \%$ of all extrapulmonary TB, and the spine is involved in $50 \%$ of these patients [1]. However, in human immunodeficiency virus (HIV)-positive patients, skeletal TB occurs in approximately $60 \%$ of patients compared with $3 \%-5 \%$ of HIV-negative patients [2].

Despite the recent advances in the diagnosis and management of spinal $\mathrm{TB}$, there remain many unaddressed issues of marked clinical significance, and urinary bladder (voiding/storage) dysfunction is one of them. Spinal cord lesions are well known to cause bladder dysfunction; they can cause neurogenic detrusor overactivity (NDO), detrusor sphincter dyssynergia (DSD), induce reflex micturition with increased detrusor leak point pressure causing incontinence and consequent renal damage if untreated [3].

Depending upon the level of spinal cord involvement, upper motor neuron/lower motor neuron (UMN/LMN) type of bladder can result. The role of urodynamic studies (UDS) in the neurogenic bladder is well established. To date, some studies have described the role of UDS in the management of neurogenic bladder following nontraumatic myelopathies (NTM) but work on TB of the spine with the neurogenic bladder is limited [4].

This study aims to evaluate patients with spinal TB presenting with lower urinary tract symptoms (LUTS) using clinical and urodynamic parameters. In addition, this study aims to evaluate the same parameters before and after spinal surgery at a follow-up of 3 months. To the best of our knowledge, this is the first study that compares the clinical and urodynamic parameters before and after surgical treatment of spinal TB.

\section{Materials and Methods}

We conducted this study in the department of urology and the department of orthopaedics at All India Institute of Medical Sciences, New Delhi. between June 2015 and June 2016. This study protocol was approved by the ethical committee of Post Graduate Research, All India Institute of Medical Sciences, New Delhi, India (IRB approval no., IESC /T-406/JULY-2015, RT-18/2015). Written informed consents were obtained. We examined patients admitted under the department of orthopaedics with a diagnosis of spinal TB with voiding dysfunction who were planned for surgery. Of note, we excluded patients with other known genitourinary pathology like stricture urethra, benign prostatic hyperplasia, or TB affecting the genitourinary tract, neurological diseases that can confound findings on urodynamic evaluation or altered sensorium patients, and patients with a history of any surgical intervention in the lower urinary tract. A total of 10 patients admitted under the department of orthopaedics with a diagnosis of spinal TB with LUTS or bladder dysfunction were evaluated. All patients underwent magnetic resonance imaging (MRI) of the spine. In addition, a detailed clinical history was obtained, and physical examination was performed. We assessed urinary symptoms by the American Urological Association (AUA) symptom score. Moreover, a baseline ultrasound scan of the kidneys and bladder with postvoid residual urine volume assessment was acquired. We collected three samples for urinary acid-fast bacilli to exclude genitourinary TB. We conducted a UDS, including cystometrogram, and pressure flow study, including electromyography, in all patients preoperatively using Laborie Aquarius TT urodynamic machine (Laborie, Mississauga, ON, Canada). As recommended by the International Continence Society, standard terminology was applied for clinical and urodynamic findings [5]. All patients underwent standard posterolateral decompression surgery for spinal TB with pedicle screw fixation; we used middle path regime in these patients. While four drug regime HRZE (isoniazid, rifampicin, pyrazinamide, and ethambutol) was administered for 4 months, two drugs HR (isoniazid and rifampicin) were administered for 14 months, making a total of 18 months. Healing was verified with contrast-enhanced MRI and the normalization of clinical symptoms. All patients were mobilized a day after surgery, and the mobilization was enhanced aggressively depending on the neurological recovery. Besides, they were given extensive chest physiotherapy. Furthermore, patients received anticholinergics and clean intermittent catheterization (CIC) was advised as per clinical and urodynamic findings. Clinical examination and urodynamic evaluation were repeated after 3 months of spinal surgery.

\section{Results}

In this study, we enrolled 10 patients (four males and six females) from June 2015 to June 2016 (Table 1). The mean age of patients was 29.7 years (range, $15-52$ years). All patients had LUTS; one patient was on an indwelling catheter for the urine retention. The mean AUA symptom score was 12.5 (range, 5-21) and 11.8 (range, 5-25) before and after spinal surgery, respectively. Of all, five patients exhibited 
Table 1. Voiding/storage dysfunction and clinical findings at presentation

\begin{tabular}{|c|c|c|c|c|}
\hline Case no. & Age $(y r) / \operatorname{sex}$ & Urinary symptoms & Clinical findings & $\begin{array}{l}\text { Tone/grade of W/ } \\
\text { level of } \mathrm{H}\end{array}$ \\
\hline 1 & $35 / F$ & PF, straining & WD, D, T & Inc/3/- \\
\hline 2 & $31 / F$ & Straining & WD, D, T & Inc/4/D4 \\
\hline 3 & $25 / \mathrm{M}$ & PF & WD, T & Inc/4/D6 \\
\hline 4 & $30 / \mathrm{M}$ & PF, history of retention, currently voiding with PF & Wheelchair bound, D, JP, V & $\operatorname{lnc} / 2 /-$ \\
\hline 5 & $17 / F$ & $\begin{array}{l}\text { PF, straining, poor bladder sensation, } \\
\text { indwelling catheter for retention }\end{array}$ & Bedridden, T, D, JP, V & Inc/0/D8 \\
\hline 6 & $15 / F$ & $\mathrm{Fr}, \mathrm{Ur}$ & WD, D, T & Inc/3/D5 \\
\hline 7 & $52 / F$ & $\begin{array}{l}\text { Loss of bladder sensation, retention with involuntary } \\
\text { leak }\end{array}$ & Walk with support, T, D, JP, V & $\operatorname{lnc} / 2 / D 6$ \\
\hline 8 & $38 / \mathrm{M}$ & $\mathrm{PF}, \mathrm{Fr}$ & WD, D, T & Inc/3/D5 \\
\hline 9 & $26 / \mathrm{M}$ & $\mathrm{Fr}, \mathrm{Ur}$ & WD, D, T & $\operatorname{lnc} / 4 /-$ \\
\hline 10 & $28 / F$ & $P F, U r$ & $W D, D, T$ & Inc/3/D5 \\
\hline
\end{tabular}

W, weakness in lower limbs; $H$, horizontal sensory loss; $F$, female; $M$, male; PF, poor flow; WD, walking difficulty; $D$, prominent deformity; T, tenderness; Inc, increased; JP, loss of joint position; $\mathrm{V}$, loss of vibration sensation; Fr, frequency; Ur, urgency.

improvement in the AUA symptom score; of these, two patients exhibited improvement of $>4$ points. While three patients exhibited no change in the AUA symptom score, two patients' AUA symptom score worsened, of which one had $>4$ points worsening. On MRI, all lesions in the spinal cord were in the dorsal cord (D1-D12, mostly D5 and D6), as detailed in Table 2. On MRI, the spinal cord lesions in all patients displayed tubercular abscess with cord compression along with a reduction in disc spaces and paradiscal involvement. In this study, patient number 5 and 7 exhibited evidence of granuloma formation, while patient 4 had substantial soft tissue swelling on MRI. Furthermore, two patients had bilateral mild hydronephrosis on preoperative ultrasound scan, which resolved on follow-up after 3 months, while one patient developed new onset bilateral mild hydronephrosis with worsening symptoms at 3-month follow-up (Table 2).

\section{Urodynamic evaluation}

\section{1) Before spinal surgery}

In this study, two patients had poor sensation of the bladder, one had early sensations, and seven patients had normal sensations. All patients had normal compliance. Detrusor overactivity (DO) was observed in two patients, while DSD was observed in four patients. In addition, both patients with DO had DSD. Overall, the mean maximum flow $\left(Q_{\max }\right)$ was $13.86 \mathrm{~mL} / \mathrm{sec}$, and the mean detru- sor pressure $\left(P_{\text {det }}\right)$ at $Q_{\max }$ was $47.77 \mathrm{~cm} \mathrm{H}_{2} \mathrm{O}$ (range, 9-96 $\mathrm{cm} \mathrm{H}_{2} \mathrm{O}$ ). We noted high-pressure voiding (HPV) in two patients. Both patients with HPV had DSD and mild upper tract changes; these two patients received anticholinergic (tolterodine $4 \mathrm{mg}$ extended release) with CIC before surgical intervention. Overall, three patients were on anticholinergic plus CIC, including the two with HPV. In addition, two patients were on CIC only. One patient was on an indwelling catheter for the retention of urine, and four patients required only observation.

\section{2) After 3 months of spinal surgery}

Two patients developed new-onset poor compliance. In addition, DO persisted in two patients, with one of them showing worsening. One patient had newly developed terminal DO with the leak. Overall, the mean $Q_{\max }$ was 12.88 $\mathrm{mL} / \mathrm{sec}$, and mean $P_{\text {det }}$ at $Q_{\max }$ was $39.47 \mathrm{~cm} \mathrm{H}_{2} \mathrm{O}$ (range, 3-103 $\mathrm{cm} \mathrm{H} \mathrm{H}_{2} \mathrm{O}$ ) at a follow-up of 3 months after surgery. One patient exhibited $P_{\text {det }}$ at $Q_{\max }$ of $103 \mathrm{~cm} \mathrm{H}_{2} \mathrm{O}$, which was $37 \mathrm{~cm} \mathrm{H}_{2} \mathrm{O}$ preoperatively. The same patient exhibited new onset poor compliance with worsening DSD. In this study, DSD persisted in four patients and worsened in one of them. HPV, which was observed in two patients in preoperative UDS, resolved in follow-up UDS but DSD persisted, and $P_{\text {det }}$ at $Q_{\max }$ decreased from $92 \mathrm{~cm} \mathrm{H}_{2} \mathrm{O}$ to $33 \mathrm{~cm} \mathrm{H} \mathrm{H}_{2} \mathrm{O}$ and $96 \mathrm{~cm} \mathrm{H}_{2} \mathrm{O}$ to $32 \mathrm{~cm} \mathrm{H}_{2} \mathrm{O}$, respectively in both patients; these patients continued with anticholinergic and CIC, as both had mild upper tract changes, 


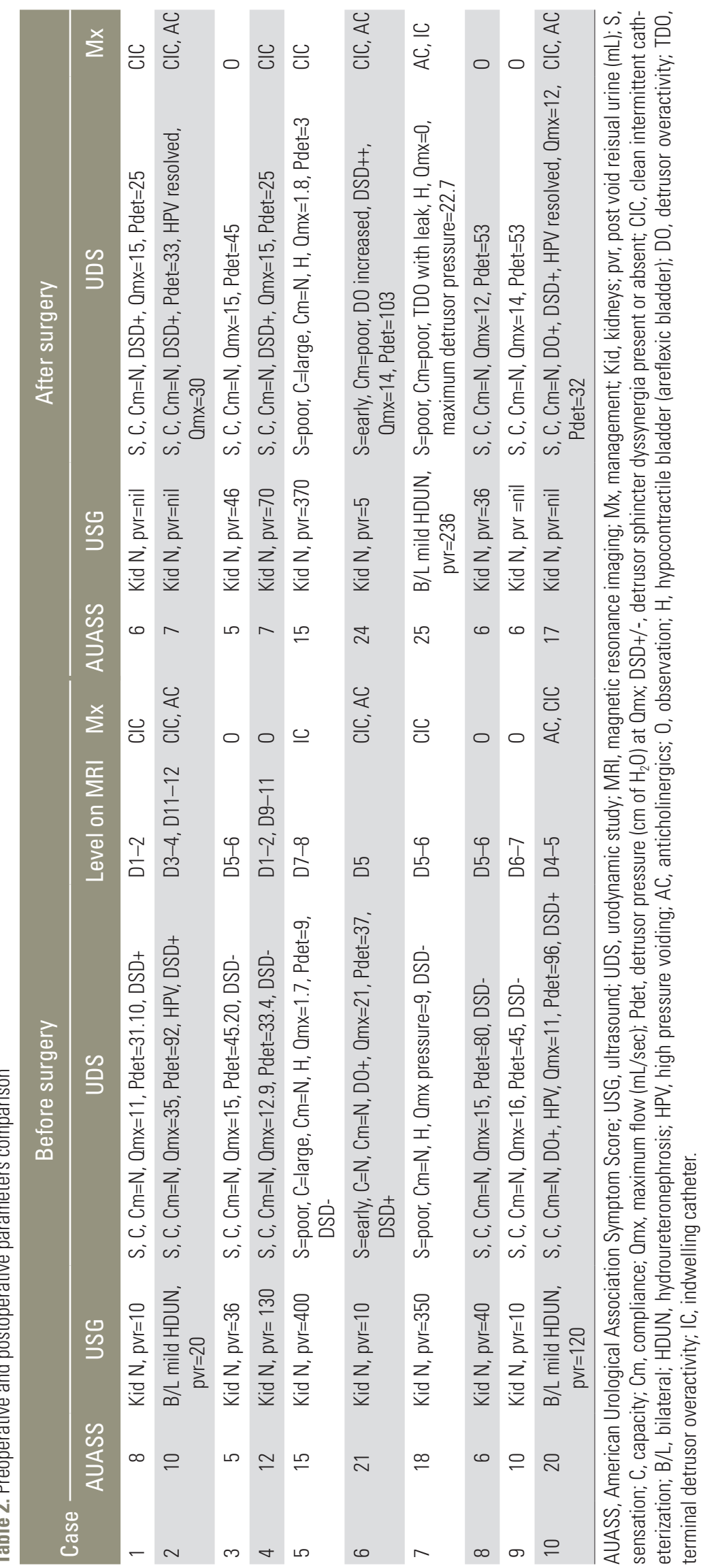


which resolved on follow-up. Postoperatively, one patient was on an indwelling catheter for worsening compliance and hydronephrosis, three patients received CIC only, and three were on CIC plus anticholinergics. However, three patients required only observation.

In this study, we could not establish any particular pattern or correlation between the level of spinal cord involvement and the detrusor function, both in the preoperative and postoperative studies (Table 2).

\section{Discussion}

Spinal cord lesions are well-known causes of bladder dysfunction. Bladder dysfunction can be of UMN type or LMN type, as per the Bors-Comarr classification depending upon the site of the lesion. These spinal lesions can result in NDO, DSD (UMN) increased detrusor leak point pressure, and consequent upper tract changes [3]. The data are sparse about bladder dysfunction resulting from Potts spine, and, to the best of our knowledge, no study to date has investigated the effects of a surgical intervention on the clinical and urodynamic profiles in these cases. Although the urodynamic and clinical profiles have been explained in the literature in traumatic myelopathies and NTM, a study of bladder dysfunction after the surgical intervention has not been reported for traumatic spinal cord injuries or spinal TB with associated neurogenic bladders.

This study reveals that patients with spinal TB presenting with LUTS could have a varied detrusor function, including completely normal UDS, areflexic bladder, DO, DO with DSD, and HPV, with variable $Q_{\max }$. In addition, the level of the spinal cord involvement on MRI did not correlate with the detrusor behavior. Similar findings were noted by Gupta and Taly [4], who observed the urodynamic profiles of 79 patients following NTM and reported that all patients had urinary complaints $(71.4 \%$ had NDO with/without DSD with lesions above D10 and 52.9\% patients with lesions between D10 and L2 had NDO with/ without DSD); in patients in the Cauda equina group, majority had underactive detrusor, and they found no significant correlation between the detrusor behavior and the NTM level. Conversely, Dong et al. [6] assessed clinical data of 30 patients with neurogenic bladder caused by intervertebral disc herniation and reported that $83.3 \%$ of patients with cervical disc herniation expressed detrusor hyperreflexia (now NDO), of which, the majority $(66.7 \%)$ were detrusor hyperreflexia with dyssynergia.
In addition, in patients with lumbar disc herniation, the majority (85.7\%) expressed detrusor areflexia; their study established a significant correlation between urodynamic results and the level of disc herniation [6].

In this study, an overall improvement in the AUA symptom score was noted (mean preoperative AUA symptom score, 12.5; postoperative AUA symptom score, 11.8), which was statistically insignificant $(p=0.55)$. In an observational study, however, Kalita et al. [7] noted significant improvement in the AUA symptom score. Kalita et al. [7] reported clinical, MRI, and urodynamic findings in 30 patients with spinal TB; of these, 15 had micturition disturbances. The authors classified urodynamic findings in these patients into detrusor areflexia and detrusor hyperreflexia with or without HPV [7]. In this study, patients received bed rest for 3 months and 4-drug antitubercular therapy (ATT). The symptoms of bladder dysfunction were assessed by the AUA symptom score, which revealed a marked improvement at 6- and 12-month follow-up. We conducted a UDS only on three follow-up patients, which revealed that detrusor areflexia was replaced by detrusor hyperreflexia with HPV in one patient and detrusor hyperreflexia persisted in the other two patients.

In this study, DO and DSD did not improve at 3-month follow-up after spinal surgical intervention and ATT. HPV resolved, but two patients developed new-onset poor compliance and worsening of DO and DSD; one of these patients developed bilateral hydronephrosis. In both the preoperative and postoperative studies, no particular pattern or correlation could be established between the level of spinal cord involvement and the detrusor function. Contrarily, Cong et al. [8] reported significant urodynamic parameters improvement after surgical intervention in cases of lumbar canal stenosis; they reported that after lumbar decompression, $43.8 \%$ of cases with abnormal preoperative urodynamic findings became normal and no new urodynamic abnormality developed. In addition, DSD was identified in eight patients preoperatively; of which, DSD persisted after decompression in five cases. Furthermore, statistically significant improvement was reported for the maximum flow rate, post-void residual urine, and maximum cystometric capacity after 6 months of surgery. Of note, no significant improvement was reported for upper tract changes.

This study has some limitations such as a small number of patients, short follow-up, and no follow-up MRI of the spine. In addition, we did not differentiate whether it 
is the surgical/medical intervention effect or the course of the disease itself affected voiding dysfunction in the follow-up period. Furthermore, the individual impact of the surgical intervention, medical management, and the course of the disease on the follow-up clinical and urodynamic outcomes needs to be evaluated.

\section{Conclusions}

Patients with spinal TB exhibiting LUTS could have different types of clinical presentation, ranging from urinary retention to features of overactive bladder; similarly, UDS findings also vary from patient to patient. UDS findings could be normal study, areflexic bladder, or an overactive bladder with or without DSD. This study demonstrates that the effect of surgical intervention on the detrusor function is not predictable, with some patients exhibiting improved while some exhibiting worsening detrusor function and upper tracts. This study concludes that a baseline and follow-up UDS is warranted for patients with spinal TB and neurogenic bladder undergoing spinal surgery, along with a close follow-up for upper tracts, and appropriate anticholinergics with or without clean intermittent self-catheterization. To the best of our knowledge, to date, no study has compared the urodynamic and clinical parameters in patients with spinal TB before and after surgical interventions. Nevertheless, further studies with larger sample size and longer follow-up are warranted to further evaluate the need and follow-up intervals.

\section{Conflict of Interest}

No potential conflict of interest relevant to this article was reported.

\section{ORCID}

Nitin Shrivastava: https://orcid.org/0000-0003-3148-2033

Prabhjot Singh: https://orcid.org/0000-0003-3562-844X

Brusabhanu Nayak: https://orcid.org/0000-0002-8047-2758

Bhavuk Garg: https://orcid.org/0000-0003-3169-6437

\section{References}

1. Polley P, Dunn R. Noncontiguous spinal tuberculosis: incidence and management. Eur Spine J 2009;18:1096-101.

2. Moon MS. Tuberculosis of the spine: controversies and a new challenge. Spine 1997;22:1791-7.

3. Gupta A, Taly AB, Srivastava A, Thyloth M. Urodynamic profile in myelopathies: a follow-up study. Ann Indian Acad Neurol 2009;12:35-9.

4. Gupta A, Taly AB. Urodynamic profile of patients with neurogenic bladder following non-traumatic myelopathies. Ann Indian Acad Neurol 2013;16:42-6.

5. Abrams P, Cardozo L, Fall M, et al. The standardisation of terminology in lower urinary tract function: report from the standardisation sub-committee of the International Continence Society. Urology 2003;61:37-49.

6. Dong D, Xu Z, Shi B, Chen J, Jiang X, Wang H. Urodynamic study in the neurogenic bladder dysfunction caused by intervertebral disk hernia. Neurourol Urodyn 2006;25:446-50.

7. Kalita J, Misra UK, Kumar G, Kapoor R. Bladder dysfunction in spinal tuberculosis: clinical, urodynamic and MRI study. Spinal Cord 2010;48:697-703.

8. Cong ML, Gong WM, Zhang QG, et al. Urodynamic study of bladder function for patients with lumbar spinal stenosis treated by surgical decompression. J Int Med Res 2010;38:1149-55. 